\title{
Mechanically triggered solute uptake in soft contact
}

\section{lenses}

Silvia Tavazzi ${ }^{l *}$, Lorenzo Ferraro ${ }^{l}$, Matteo Fagnola, Federica Cozza ${ }^{l}$, Stefano Farris ${ }^{2}$, Simone Bonetti $^{1}$, Roberto Simonutti ${ }^{1}$, Alessandro Borghesi ${ }^{1}$

${ }^{1}$ Materials Science Department, University of Milano Bicocca, Via Cozzi 55,

$$
\text { I-20125 Milan, Italy }
$$

${ }^{2}$ DeFENS, Department of Food, Environmental and Nutritional Sciences, Packaging DivisionUniversity of Milan, Via Celoria 2, I-20133, Milan, Italy

*E-mail silvia.tavazzi@unimib.it, Tel. +39 02 64485035, Fax +39 0264485400 


\section{ABSTRACT}

Molecular arrangement plays a role in the diffusion of water and solutes across soft contact lenses. In particular, the uptake of solutes in hydrated contact lenses can occur as long as free water is available for diffusion. In this work, we investigated the effect of mechanical vibrations of low frequency $(200 \mathrm{~Hz})$ on the solute uptake. Hyaluronan, a polysaccharide of ophthalmic use, was taken as example of solute of interest. For a specific hydrogel material, differential scanning calorimetry experiments showed that a large fraction of the hydration water accounted for looselybound water, both before and after one week of daily-wear of the lenses. The size (of the order of magnitude of few hundreds of nanometers) of hyaluronan in aqueous solution was found to be less than the size of the pores of the lens observed by scanning electron microscopy. However, solute uptake in already-hydrated lenses was negligible by simple immersion, while a significant increase occurred under mechanical vibrations of $200 \mathrm{~Hz}$, thus providing experimental evidence of mechanically triggered enhanced solute uptake, here attributed to the release of interfacial looselybound water. Also other materials were taken into consideration. However, the effectiveness of mechanical vibrations for hyaluronan uptake is restricted to lenses containing interfacial looselybound water, which is expected to be bound to the polymer with bonding energies (of the order of magnitude of 10-100 J/g) compatible with the energy input supplied by the vibrations.

KEYWORDS: water/polymer interface; contact lenses; mechanical vibrations; hyaluronan; controlled release 


\section{INTRODUCTION}

Soft contact lenses (CLs) are widely used for the correction of refractive errors of the eye. In recent years, the lens absorption of various molecules and macromolecules has been increasingly taken into consideration [1-6]. One reason to study the CL absorption of various molecules is the presence of molecules and macromolecules both in the tear fluid and in the CL maintenance solutions. Another reason is the interest in CLs as reservoir and release systems of drugs and macromolecules for specific applications, in contrast with most of conventional ophthalmic formulations, which require direct application of doses at high concentrations due to the large and rapid draining of the drug through the lachrymal duct, with also possible undesirable effects.

Hyaluronan (also called hyaluronic acid, HA) is one of the molecular species of special interest for its ophthalmic advantages. HA is a polysaccharide present in many human tissues (e.g. connective, epithelial, and neural), with applications in medicine, pharmacy, biotechnology, and ophthalmology [7-14]. The HA uptake in CLs could also improve the comfort performance of the CLs due to its high hydrophilicity; in addition, HA can prevent the adsorption in the polymeric network of detrimental substances, which could possibly give rise to several problems during the wear, such as cytotoxicity and ocular irritation. In this respect, the HA uptake capability of a CL and the possibility to reload the CL in its hydrated state during the wear period are relevant aspects. HA can also be considered a model system to discuss the uptake capability of the CLs and to investigate the uptake mechanisms of ocular drugs, as potential controlled-release delivery platforms [1-6]. A recent work was reported on the HA distribution and penetration depth inside a siloxane-hydrogel CL [15]. HA was found to fill the CL pores, which could be seen by electronic and fluorescence microscopic techniques on the surface of the CL (Filcon V). However, the HA was found to reach a penetration depth of the order of 5-10 $\mu \mathrm{m}$, to be compared with the much larger thickness $(\sim 100$ $\mu \mathrm{m})$ of the whole CL. This experimental evidence suggested that a better understanding of the uptake of a solute in CLs can be achieved by investigating the compatibility between CL (in terms 
of morphology and porosity) and solute (in terms of size of the molecules and/or size of possible aggregates of such molecules).

Another very important aspect for the uptake of solutes in hydrated CLs is related to the properties of the hydration water. For example, a detailed study was reported by Tranoudis and Efron, who examined the state of water in several soft CLs [16]. The authors found that low water-content CLs have approximately the same amount of bound water as those with much higher water-content. The excess of water in the latter ones is represented by loosely-bound water at the water-polymer interface and, eventually, free water in the bulk. Based on the typical classification of the state of water in hydrogels [17], tightly-bound water has a direct hydrogen bonding with the polar groups of the polymer. Free water does not interact with the polymer and has hydrogen-bonding characteristic of pure water. Loosely-bound water is interfacial and remains in liquid state slightly below the normal freezing temperature (with negative freezing temperature between $0^{\circ} \mathrm{C}$ and $-10^{\circ} \mathrm{C}$ ). A typical example of interfacial loosely-bound water is represented by water in swollen polymers [16]. Free water is able to participate to diffusion, so that it is relevant for solute uptake in CLs exposed to solutions. In this respect, loosely-bound water could be released, thus becoming free, if some energy is provided of the order of, at least, $10-100 \mathrm{~J} / \mathrm{g}$, since this value is approximately the expected binding energy of loosely-bound water to the polymer [18.19]. This bonding energy is much lower than the typical strength of hydrogen bonds and also much lower than the expected bonding energy of tightly-bound water to the polymer [20]. For this reason, triggers could be used as energy input for the release of loosely-bound water from CLs, which in turn would promote both diffusion and solute uptake. An example of external trigger is given by the application of mechanical vibrations. In the literature, the effects of mechanical vibrations were discussed as far as the fracture of the HA backbone is concerned under sonication at a relatively very high frequency ( 2 $\left.10^{4} \mathrm{~Hz}\right)$ and power $(7.5 \mathrm{~W})[21]$.

This paper is aimed at investigating the effects of mechanical vibrations of about $150 \mathrm{~mW}$ and much lower frequency $(200 \mathrm{~Hz})$ than reported in the literature on the uptake of HA in soft CLs in 
their hydrated state, both before and after one week of wear. A preliminary combined characterization was first performed. On one hand, some CLs were characterized by scanning electron microscopy (to gather information on their structure and porosity) and by differential scanning calorimetry (to elucidate the state of the hydration water), both before and after wear. On the other hand, aqueous solutions of HA were characterized by dynamic light scattering to deduce the size distribution of HA particles/aggregates. Then, the quantity of absorbed solute per lens in solution was determined both without and under mechanical vibrations. Results are discussed on the basis of the properties of both the CLs and the hydration water and on the basis of the energy provided by the vibrations, which was found to be sufficient for the release of interfacial looselybound water and for restoring water diffusion mechanisms.

\section{MATERIALS AND METHODS}

Three types of commercial CLs were taken into consideration: (i) Methafilcon 1B (Safilens, IV FDA group) [13], a hydroxyl-ethyl-methacrylate (HEMA) copolymer and methacrylic acid (MA), (ii) Filcon V (Safilens, I FDA group) [15] containing dimethyl-acrylamide, tris-trimethylsilys, poly(dimethyl-siloxane)-dimethacrylate, ethyleneglycol-dimethacrylate, phthalocyanine copper, (iii) Senofilcon A (Johnson\&Johnson, I FDA group) containing polydimethyl-siloxane (PDMS), N,Ndimethylacrylamide (DMA), polyvinyl-pyrrolidone, and HEMA. Only in one case, the CLs were rinsed in saline aqueous solution $(0.9 \% \mathrm{NaCl})$ purchased from Alcon Italia, to compare their hydration volume in either saline solution or deionized water. In all other cases, the CLs were rinsed and hydrated in deionized water.

Scanning electron microscopy (SEM) micrographs of the CL surfaces were obtained with the goal of acquiring information on the structure and porosity of the CLs. The CLs were examined using a LEO 1430 (Carl Zeiss, Oberkochen, Germany) operating at $20 \mathrm{kV}$ and $3 \cdot 10^{-3}$ Torr. Before analysis, 
samples were freeze-dried $\left(-55^{\circ} \mathrm{C}, 0.63\right.$ mbar $\times 24$ hours $)$ using an ALPHA 1-2 LDplus freeze dryer (Martin Christ, Osterode am Harz, Germany) and then sputter-coated with gold to a thickness of approximately $10 \mathrm{~nm}$ using a Semprep 2 sputter coater (Nanotech Ltd., Prestwick, UK) at 10 mA.

Melting endotherms of water in water-hydrated CLs were determined by differential scanning

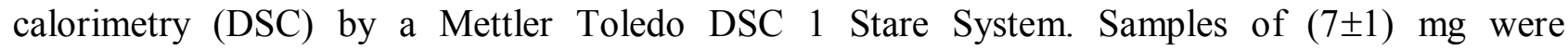
characterized with the following method: (i) 3 min isotherm $-40^{\circ} \mathrm{C}$, (ii) $-40^{\circ} \mathrm{C}$ to $+30^{\circ} \mathrm{C} @ 5^{\circ} \mathrm{C} / \mathrm{min}$. All segments were driven under $80 \mathrm{~mL} / \mathrm{min} \mathrm{N}_{2}$ flux. Before analyses, CLs were lightly blotted with tissue to remove excess surface water and then mechanically sealed in $40 \mu \mathrm{L}$ aluminum pan. To achieve a high reproducibility of results for materials prone to fast dehydration (i.e. avoiding water evaporation before scanning), the blotted samples were prepared following the procedure of Tranoudis et al. [16]. By measuring temperature and heat flow associated with specimen transition, quantitative data were obtained on endothermic (heat absorption) processes.

Bacillus subtilis derived HA (MW $800 \mathrm{kDa}$ ) was purchased from Novozyme. HA solutions in deionized water were prepared with different concentrations and their refractive index was measured using a refractometer Atago RX5000- $\alpha$ (resolution $10^{-5}$ ) at $(20.00 \pm 0.01)^{\circ} \mathrm{C}$. The measured refractive index of reference HA solutions with concentrations ranging from 0.05 to $0.3 \% \mathrm{~W} / \mathrm{w}$ is reported in Fig. 1. The experimental data are well described by a linear dependence of the refractive index on the HA concentration $(\mathrm{R}>0.999)$. The result of the linear regression is also indicated in Fig. 1 by a line, which represents the calibration curve for the following analyses.

The HA solutions were also characterized by dynamic light scattering (DLS) at $25^{\circ} \mathrm{C}$ by a Malvern Instruments Nano Series Zetasizer optical unit (Continuous Wave $4 \mathrm{~mW}$ He-Ne class 1 internal laser operating at $632.8 \mathrm{~nm}$, measurement angles at $13^{\circ}$ and $175^{\circ}$ with Avalanche photodiode detector with Q.E. $>50 \%$ at $633 \mathrm{~nm}$ ). 
Both dehydrated and water-hydrated CLs of the different materials were exposed to HA solution following, in each case, the steps described below:

(i) The weight of one dehydrated CL was found by averaging on several measurements.

(ii) The weight of one water-hydrated CL was found by averaging on several measurements. The weight of the water contained in a CL was obtained by subtracting the weight of the CL in the hydrated and dehydrated forms. For each CL, the ratio (defined as EWC) between the weight of the water contained in a CL and the total weight of the hydrated CL was also calculated.

(iii) In a glass vial, $10 \mathrm{CLs}$ of the same material were exposed together to $1 \mathrm{~g}$ of $0.2 \% \mathrm{w} / \mathrm{w} \mathrm{HA}$ aqueous solution.

(iv) The 10 CLs were extracted from the vial after a fixed time span (varying from about 10 minutes to eight hours). To minimize the amount of droplets of residual solution on the surface of the CLs, they were dripped for $10 \mathrm{~s}$ during the extraction from the vial.

(v) The weight of the extracted hydrated CLs was measured.

(vi) The weight of the residual solution in the vial and its refractive index were measured. The refractive index was then converted into a HA concentration value using the calibration curve (Fig. 1).

(vii) The weight of absorbed HA per one lens $\left(\mathrm{W}_{\mathrm{abs}}\right)$ was evaluated as 1/10 of the difference of the HA content (i.e. HA weight) in the vial before and after the loading procedure. These HA contents were deduced by taking into consideration the initial concentration and weight of the loading solution and the final concentration and weight of the vial solution after removing the CLs. When CLs were exposed to the loading solution in their hydrated form, no significant differences were typically found in the weights of the vial solution before and after the CL loading. On the contrary, the CL exposure in their dehydrated form was obviously found to significantly decrease the weight of residual solution compared to the initial weight, due to CL hydration during the procedure. 
In a second procedure, the loading of HA (step (iii)) was carried out under mechanical vibrations. For this purpose, the vial was substituted with a plastic vessel anchored to a generator of mechanical vibrations (provided by Safilens, Italy). The loading of HA was performed by exposing the water-hydrated CLs to the HA aqueous solution in the vessel and applying mechanical vibrations of $200 \mathrm{~Hz}$ and $150 \mathrm{~mW}$ for 60 seconds. Except step (iii), all the previous steps of the loading procedure were carried out as previously described.

As far as the step (vi) of the procedure is concerned, the variation of refractive index was attributed to a variation of the HA concentration, as a consequence of HA absorption in the $10 \mathrm{CLs}$. A change of refractive index due to the release in solution of residual chemical components from the CLs could be excluded. Indeed, some preliminary analyses were performed exposing the 10 CLs to pure deionized water. In this case, also when applying the vibrations, no significant variations of the refractive index were detected.

When comparing different groups (different materials or different applied procedures), statistical significance of differences among the mean of the measured values was evaluated by Student's statistic (considering significant difference if the probability value $p$ was lower than 0.05 ).

\section{RESULTS}

Figures 2, 3, and 4 show typical SEM micrographs taken from the surface of different CLs. Empty pores are clearly seen in Fig. 2 (Methafilcon 1B), with diameters as large as several micrometers. These pores appear well defined, in particular in the micrograph reported in panel $2 b$. As shown in panel 2a, some inhomogeneity in the pore distribution was found on the CL surface. A possible origin of this inhomogeneity could be related to the manufacturing process, but further investigation are required to better understand it. The surface of the Filcon V material was also found to show 
relatively large pores, with diameters of the order of magnitude of the micrometer (Fig. 3). As mentioned before, detailed information on the properties of this material are reported elsewhere [15]. No significant pores were observed for the Senofilcon A CLs (Fig. 4), as also found for other materials, such as, for example, Comfilcon A (see Fig. S1 of the Supplementary Material).

Besides the porosity and structure, also the water content of the CLs was investigated. EWC was measured both in deionized water and in $\mathrm{NaCl}$ solutions to assess possible differences in the $\mathrm{CL}$ swelling properties. Figure 5 shows the total EWC (free + loosely-bound + tightly-bound water) as a function of the $\mathrm{NaCl}$ concentration of the hydration solution. In deionized water, the properties of the hydration water were also investigated by DSC. Figure 6 shows the DSC curves for both new (left) and worn (right) CLs. Table I summarizes the data obtained by weight (i.e. total EWC) measurements and endothermic DSC curves in Fig. 6. In the latter figures, two peaks can be observed: one sharp peak centred at $0^{\circ} \mathrm{C}$ (peak A) and a second broader peak (peak B) in the region between $-2^{\circ} \mathrm{C}$ and $-10^{\circ} \mathrm{C}$. Peak A corresponds to the free water content of CLs, while peak B corresponds to the loosely-bound water [16]. The free water content (given in EWC in Table I) was calculated comparing the area under the melting band centered at about $0^{\circ} \mathrm{C}$ with the latent heat of fusion of water $(340.6 \mathrm{~J} / \mathrm{g}$ ). The loosely-bound water content (given in EWC in Table I) was calculated in a similar way by considering the melting bands between about $-2^{\circ} \mathrm{C}$ and $-10^{\circ} \mathrm{C}$. All data in Table I are given within an estimated error of about $5 \%$, the errors deriving mainly from the dripping of the hydrated CLs before both the weight and the DSC measurements and from the selection of the integration range in the DSC curve to distinguish free and loosely-bound water.

As far as the characterization of the loading solution is concerned, the HA aqueous solutions were found by DLS to show particles with typical hydrodynamic diameters of the order of $(20 \pm 5) \mathrm{nm}$ and $(120 \pm 15) \mathrm{nm}$ at concentrations ranging from $0.01 \% \mathrm{~W} / \mathrm{w}$ to $0.1 \% \mathrm{~W} / \mathrm{w}$, independent of the concentration (Supplementary Material, Fig. S2). The larger diameter is attributed to the size of one hydrated HA chain (800 $\mathrm{kDa}$ molecular weight), while the smaller value is attributable to HA chains, which are not well-hydrated. At the concentration of interest $(0.2 \% \mathrm{w} / \mathrm{w})$, DLS results are 
expected to be influenced by multiple scattering and by the relatively high viscosity of the solution. Therefore, the lack of HA clusters larger than few hundreds of nanometers in $0.2 \% \mathrm{w} / \mathrm{w}$ solution was inferred by measuring the refractive index before and after filtering it through pores of $0.2 \mu \mathrm{m}$, since no substantial differences were detected.

As far as the uptake of HA is concerned, the amount of absorbed HA in the CLs was evaluated by the seven steps as described in the previous section. The obtained weight of absorbed HA per lens $\left(\mathrm{W}_{\mathrm{abs}}\right)$ was then compared to a reference HA weight value per one lens $\left(\mathrm{W}_{\mathrm{ref}}\right)$ obtained by assuming that the CL is completely hydrated by HA solution at the osmotic equilibrium with the solution in the vial/vessel. The HA content per $\mathrm{CL}$ was thus described by the ratio $\mathrm{W}=\mathrm{W}_{\mathrm{abs}} / \mathrm{W}_{\text {ref, }}$, which represents the fraction of absorbed solute compared to the limiting osmotic equilibrium value. The W values reported in Table II are the average values obtained for the different samples, both when exposed to the HA solution in the dehydrated state and when exposed to the HA solution in the water-hydrated state, either without or under mechanical vibrations. Statistical significant difference between the mean values of the corresponding groups was evaluated. The $p$ values of the Student's statistics are reported in the Supplementary Material (Table S-I). The values in Table II are the ratios $\mathrm{W}$ between $\mathrm{W}_{\mathrm{abs}}$ and $\mathrm{W}_{\text {ref. }}$ The corresponding $\mathrm{W}_{\mathrm{abs}}$ values of absorbed HA per CL are reported in the Supplementary Material (Table S-II) for the Methafilcon 1B material.

\section{DISCUSSION}

The first peculiarity of Methafilcon 1B CLs was found by SEM analysis. The hydrated material showed very large pores with variable diameters up to several micrometers (Fig. 2), much larger than the HA hydrodynamic diameter in solution (about 20 and $120 \mathrm{~nm}$, as measured by DLS). The pores are typically observed both in the center and in the periphery of the CLs, notwithstanding 
some inhomogeneity in their distribution, as can be seen in Fig. 2 (panel a). Since these pores are filled by water, they do not represent a limit for light transmission by the CLs, due to the relatively low contrast of refractive index between the polymer and water. For Filcon V CLs, Fig. 3 shows similar structures as recently reported elsewhere for the same material [15], where the presence of a porous layer close to the CL surface was discussed. For Senofilcon A, only few pores were rarely observed in the peripheral region of the CLs. The typical SEM micrographs are well represented by Fig. 4. Another example is reported in Fig. S1 of the Supplementary Material for a Comfilcon A CL, where no pores are detected, within the resolution of the image.

Another important feature of Methafilcon 1B is its water-swelling capability. Among those investigated, Methafilcon $1 \mathrm{~B}$ is the only material which strongly increases its EWC from $0.9 \%$ $\mathrm{NaCl}$ aqueous solution to deionized water (Fig. 5), up to EWC $\sim 70 \%$ w/w. From DSC analyses, a significant fraction of this water was found to be free (new CL: EWC $=15.9 \%$, worn CLs: EWC = $13.1 \%$ and $\mathrm{EWC}=17.8 \%$ ) or loosely-bound (new CL: $\mathrm{EWC}=18.8 \%$, worn CLs: $\mathrm{EWC}=11.2 \%$ and $\mathrm{EWC}=10.9 \%$ ), in contrast to the other materials where the sum of free and loosely-bound water represents few $\%$ in terms of EWC, within an experimental error of about $5 \%$. The presence of loosely-bound water is typically expected in water-swollen polymers, as the case of Methafilcon 1B in aqueous phase. The EWC value for loosely-bound water in new Methafilcon 1B CLs (18.8\%, i.e. $\sim 8 \mathrm{mg}$ ) is also in reasonable agreement, within the limits of this comparison, with the expected volume of interfacial water. Indeed, based on SEM results, a distribution of pore size is expected. Typically, large pores are less numerous than small ones [15], so that a very crude approximation of the mean size of the pores is reasonably $0.2 \mu \mathrm{m}$. This approximated value allows to calculate the approximated mean content of water in one pore $\left(\sim 410^{-12} \mathrm{mg}\right)$ and, in turn, the approximated number of pores in a CL $\left(\sim 710^{12}\right)$ as the ratio between the total amount of water in one CL $(\sim 30$ mg) and the mean content of water in one pore. The approximated diameter of one pore and the number of pores allow to estimate the expected exposed internal area of the pores, which is expected to be loosely-bound to water. This area in $\sim 10^{12} \mu \mathrm{m}^{2}$. To obtain $8 \mathrm{mg}$ of loosely-bound 
water (as by DSC analyses), the thickness of the interfacial layer of water should be of the order of magnitude of few nanometers, as expected [22] for loosely-bound water.

Also the dependence of the $\mathrm{EWC}$ on $\mathrm{NaCl}$ concentration (Fig. 5) can be interpreted as a consequence of the presence of a large fraction of interfacial loosely-bound water in Methafilcon 1B. Indeed, adding an ionic salt reduces the number of available sites of the hydrogel for bonding with water molecules, thus significantly reducing the amount of loosely-bound water. This strong dependence of EWC as a function of salt concentration was not detected for the other materials, where water was mainly tightly-bound (see Table I).

Also the results obtained by simple exposure of water-hydrated Methafilcon 1B CLs to the HA solution can be interpreted as a consequence of the properties of the hydration water. Indeed, the absorbed HA per CL was found to be low without vibrations, i.e. few $\mu$ g per CL (Table S-II), which corresponds to $\mathrm{W} \sim 9 \%$ of the loading limit at the osmotic equilibrium (Table II). This result can be interpreted as a consequence of the scarce water diffusion by simple immersion. Since $\mathrm{W} \sim 9 \%$ is the percentage of absorbed solute compared to the limiting osmotic equilibrium value, the percentage of free water in the CLs is also expected to be of the order of $9 \%$, which is in reasonable agreement, within the experimental errors, with the DSC results (15.9\% of free water). The loading under mechanical vibrations was significantly different. In this case, even if exposed to the HA solution in their water-swollen state, Methafilcon 1B CLs absorbed a non-negligible amount of HA, i.e. $(44.5 \pm 10.6) \mu \mathrm{g}$ per lens corresponding to approximately $\mathrm{W}=57.4 \%$ of the limiting amount at the osmotic equilibrium (Table II and Table S-II). Such a quantity indicates that vibrations induce the solute uptake. A possible explanation is the release of loosely-bound water under vibrations, making it free and available for diffusion. In particular, the percentage of solute uptake is expected to be comparable to the percentage of free water and $\sim 57 \%$ is in reasonable agreement with the sum of free and loosely-bound water in Methafilcon CLs $(\sim 35 \%)$, within the experimental errors of both DSC and uptake experiments. The increase of the HA uptake under vibrations is not attributable to 
possible decrease of the HA hydrodynamic diameter under vibrations, which could favor the uptake. Indeed, the HA hydrodynamic diameter in solution was measured to be much lower than the observed diameter of the pores of the CL also without any vibration, as discussed above. The explanation in terms of release of loosely-bound water under vibrations is supported by some considerations of the energies provided to the system. Mechanical vibrations of $150 \mathrm{~mW}$ provided an energy of about $10 \mathrm{~J}$ per minute to the $10 \mathrm{CLs}$ in the vessel, which was available for the release of the loosely-bound water. We already discussed that the loosely-bound water was deduced from DSC to be a fraction $(18.8 \%$, i.e. $\sim 8 \mathrm{mg}$ per lens) of the CL hydration. Therefore, in one cycle the available energy provided by the vibrations is of the order of $100 \mathrm{~J} / \mathrm{g}$. This relatively low energy is a reasonable value for the bonding energy of the loosely-bound water to the polymer [18.19]. Also after one week of daily-wear of the Methafilcon 1B CLs, worn CLs show the same endothermic peak shape as the new CL (Fig. 6), but the involved enthalpy is lower, so that a slight decrease of the content of loosely-bound water was detected (Table I). This can be a consequence of possible obstruction of pores, as a consequence of wear []. However, also worn CLs were found to exhibit a relatively large fraction of loosely-bond water, thus indicating the expected prolonged effectiveness of the mechanical vibrations acting on loosely-bound water, also after one week of wear.

Based on the previous considerations, the mechanism of HA uptake in water-hydrated Methafilcon 1B CLs is mechanically triggered diffusion. However, the effect is limited to CLs with sufficiently large pores and to CLs hydrated by a large fraction of loosely-bound water, due to the relatively low energy provided by the vibrations. Interestingly, another material was recently reported to show a relatively large fraction of loosely-bound water, i..e Nelfilcon (II FDA group) [23] and the effectiveness of vibrations on solute uptake could also be investigated for this material, after a morphological characterization.

Another explanation for the enhanced solute uptake under vibration could also be dispersionassisted HA uptake. Through dispersion, vibrations could accelerate the HA uptake compared to the static case. However, without any vibration, the HA uptake in the Methafilcon 1B CLs in solution 
was measured to be negligible also after several hours $(8 \mathrm{~h})$. For this reason, we believe that the enhanced uptake is not dispersion-assisted, but triggered by the release of loosely-bound water, which becomes free and participate to diffusion only under vibrations.

A different scenario was found when the CLs were exposed to the HA loading solution in their dehydrated state. In these cases, HA solution invaded the polymeric network and the HA uptake was relatively high for Methafilcon 1B and also for the other investigated materials (Table II). To obtain detailed information on the dynamics of this swelling-controlled uptake, the time evolution of the hydration should be investigated in the restricted time interval where the hydration is significantly less than its equilibrium value [24-28]. In our case, the maximum value was already reached at the shortest measured time (10 minutes), so that a detailed analysis of the characteristics of the swelling process was not performed.

\section{CONCLUSIONS}

Experimental evidence of enhanced solute (hyaluronan) uptake in solution is provided for hydrogel CLs under mechanical vibrations of relatively low power $(150 \mathrm{~mW})$ and frequency $(200 \mathrm{~Hz})$ for 60 seconds. Only one material out of three showed this enhancement. The pores of this CL were found to be much larger than the HA size in aqueous solution. However, the solute uptake in solution by simple exposure was relatively low, due to the low content of free water available for diffusion. A large fraction of the hydration water was found to be loosely-bound and our interpretation of the increase of solute uptake under mechanical vibrations is the release of this loosely-bound water under vibrations, thus becoming free and participating to diffusion. Also worn CLs of the same material were found to exhibit a relatively large fraction of loosely-bond water, thus indicating the expected prolonged effectiveness of the mechanical vibrations acting on loosely-bound water, also after one week of daily wear. 


\section{REFERENCES}

1 Hiratani H.; Alvarez-Lorenzo C. Timolol uptake and release by imprinted soft contact lenses made of N,N-diethylacrylamide and Methacrylic acid. J. Controll. Rel. 2002, 83, 223 - 230.

2 Karlgard C.C.S.; Wong N.S.; Jones L.W.; Moresoli C. In vitro uptake and release studies of ocular pharmaceutical agents by silicon-containing and p-HEMA hydrogel contact lens materials. Intern. J. Pharm. 2003, 257, $141-151$.

3 Gulsen, D.; Chauhan, A. Ophthalmic Drug Delivery through Contact Lenses. Invest. Ophthalmol. Vis. Sci. 2004, 45, 2342 - 2347.

4 Hiratani H.; Fujiwara A.; Tamiya Y.; Mizutani Y.; Alvarez-Lorenzo C. Ocular release of timolol from molecularly imprinted soft contact lenses. Biomater. 2005, 26, 1293 - 1298.

5 Verestiuc, L.; Nastasescu, O.; Barbu, E.; Sarvaiya, I.; Green, K.L.; Tsibouklis, J. Functionalized Chitosan/NIPAM (HEMA) Hybrid Polymer Networks as Inserts for Ocular Drug Delivery: Synthesis, in Vitro Assessment, and in Vivo Evaluation. J. Biomed. Mater. Res. A 2006, 77A, $726-735$.

6 Xinming L.; Yingde C.; Lloyd A.W.; Mikhalovsky S.V.; Sandeman S.R.; Howel C.A.; Liewen L. Polymeric hydrogels for novel contact lens-based ophthalmic drug delivery systems: A review. Cont. Lens \& Ant. Eye 2008; 31, 57 - 64.

7 Kogan G.; Soltés L.; Stern R.; Gemeiner P. Hyaluronic acid: a natural biopolymer with a broad range of biomedical and industrial applications. Biotechnol. Lett. 2007, 29, 17 - 25.

8 Polack F.M.; McNiece M.T. The treatment of dry eyes with Na hyaluronate-Healon. Cornea 1982, $1,133-136$.

9 Limberg M.B.; McCaa C.; Kissling G.E.; Kaufman H.E. Topical application of hyaluronic acid and chondroitin sulfate in the treatment of dry eyes. Am. J. Ophthalmol. 1987, 103, 194. 
10 Weeks A.; Subbaraman L.N.; Jones L.; Sheardown H. Physical entrapment of hyaluronic acid during synthesis results in extended release from model hydrogel and silicone hydrogel contact lens materials. Eye \& Contact lens 2013, 39, 179.

11 Van Beek M.; Jones L.; Sheardown H. Hyaluronic acid containing hydrogels for the reduction of protein adsorption. Biomater. 2008; 29, 780.

12 Van Beek M.; Weeks A.; Jones L.; Sheardown H. Immobilized hyaluronic acid containing model silicone hydrogels reduce protein adsorption. J. Biomater. Sci. Polym. Ed. 2008, 19, 1425.

13 Leonardi A.; Decastello N.; Brun P.; Violato D.; Fregona I. Release of Hyaluronic Acid From Filconlb Contact Lens Increases Tolerability and Performance. ARVO's 2007 Annual Meeting, May 6-10 2007, Fort Lauderdale, Florida.

14 Fagnola M.; Pagani M.P.; Maffioletti S.; Tavazzi S.; Papagni A. Hyaluronic acid in hydrophilic contact lenses: Spectroscopic investigation of the content and release in solution. Cont. Lens \& Anter. Eye. 2009, 32, 108 - 112.

15 Tavazzi S., Tonveronachi M., Fagnola M., Cozza F., Ferraro L., Borghesi A., Ascagni M., Farris S., Wear effects on microscopic morphology and hyaluronan uptake in siloxanehydrogel contact lenses. J. Biomed Mater Res B (accepted).

16 Tranoudis I.; Efron N. Water properties of soft contact lens materials. Cont. çens \& Anter. Eye 2004, 27, 193 - 208.

17 Pedley D.G.; Tighe B.J. Water binding properties of hydrogel polymers for reverse osmosis and related applications. Br. Polym. J. 1979, 11, $130-136$.

18 Silverstein K.A.T., Haymet A. D. J., Dill K.A. The strength of hydrogen bonds in liquid water and around nonpolar solutes. J. Am. Chem. Soc. 2000, 122, 8037.

19 Kulthe V.V., Chaudhari P.D., Drug Resinates an Attractive Approach of Solubility Enhancement of Atorvastatin Calcium. Ind. J. Pharm. Sci. 2013, 75, 523. 
21 Koubo K., Nakamura, T.; Takagaki, K.; Yoshida, Y.; Endo. M. Depolymerization of hyaluronan by sonication, Glycoconjugate $J$. 1993, 10, 435 - 439 .

22 review

23 K. Krysztofiak, A. Szyczewski, Study of dehydration and water states in new and worn soft contact lens materials. Optika applicata 2014, 44, 237.

24 Kim, S.W.; Bae, Y.H., Okano, T. Hydrogels: swelling, drug loading, and release, Pharm. Res. 1992, 9, $283-290$.

25 Chen, J.; Park, H.; Park, K. Synthesis of superporous hydrogels: Hydrogels with fast swelling and superabsorbent properties, J. Biomed. Mater. Res. 1999, 44, 53 - 62.

26 H. Omidian, et al., Advances in superporous hydrogels, Journal of Controlled Release 102 (2005) 3.

27 Wang, J.; Wu, W.; Lin, Z. Kinetics and Thermodynamics of the Water Sorption of 2Hydroxyethyl Methacrylate/Styrene Copolymer Hydrogels, J. Appl. Polym. Sci. 2008, 109, $3018-3023$.

28 Ganji1, F.; Vasheghani-Farahani, S.; Vasheghani-Farahani1, E. Theoretical Description of Hydrogel Swelling: A Review, Iran. Polymer J. 2010, 19, 375 - 398. 


\section{FIGURE CAPTIONS}

Figure 1. Calibration line. Measured refractive index of HA aqueous solutions with different concentrations and line obtained by linear fitting of the experimental data. Error bars indicate the std dev obtained by repeating the measurements at least 6 times for each concentration.

Figure 2. Methafilcon 1B. SEM micrographs of Methafilcon 1B CLs.

Figure 3. Filcon V. SEM micrograph of a Filcon V CL.

Figure 4. Senofilcon A. SEM micrograph of a Senofilcon A CL.

Figure 5. Salt effect on swelling. Hydration (weight of the hydration solution divided by the total weight of the hydrated $\mathrm{CL}$ ) as a function of the $\mathrm{NaCl}$ concentration in the aqueous hydration solution for the different types of CLs. The error bars represents $5 \%$ of the measured value. This error mainly derives from the dripping of the hydrated CLs before the measurement of their weight.

Figure 6. Free and loosely-bound water. Endothermic DSC curves for a new water-hydrated Senofilcon A CL (red line, left panel), a new water-hydrated Filcon V CL (blue line, left panel), a new water-hydrated Methafilcon 1B CL (black lines, both left and right panels), and two waterhydrated Methafilcon 1B CLs after one week of daily-wear by two different subjects (green and red lines, right panel). 